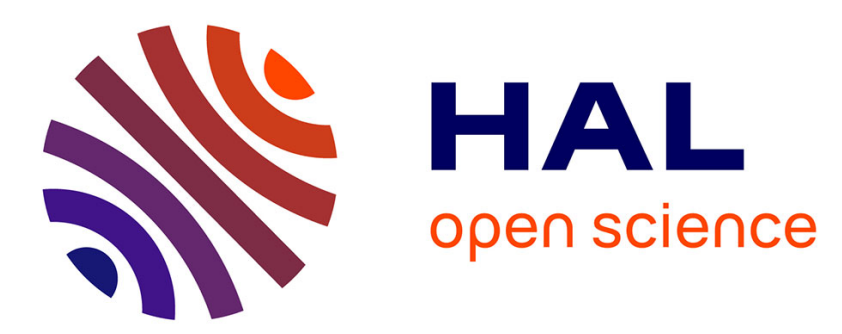

\title{
Finding Maximal Common Subgraphs via Time-Space Efficient Reverse Search
}

\author{
Alessio Conte, Roberto P Grossi, Andrea Marino, Luca P Versari
}

\section{To cite this version:}

Alessio Conte, Roberto P Grossi, Andrea Marino, Luca P Versari. Finding Maximal Common Subgraphs via Time-Space Efficient Reverse Search. COCOON 2018 - International Computing and Combinatorics Conference, Jul 2018, Qing Dao, China. pp.328-340, 10.1007/978-3-319-94776-1_28 . hal-01964709

\section{HAL Id: hal-01964709 \\ https://hal.inria.fr/hal-01964709}

Submitted on 23 Dec 2018

HAL is a multi-disciplinary open access archive for the deposit and dissemination of scientific research documents, whether they are published or not. The documents may come from teaching and research institutions in France or abroad, or from public or private research centers.
L'archive ouverte pluridisciplinaire HAL, est destinée au dépôt et à la diffusion de documents scientifiques de niveau recherche, publiés ou non, émanant des établissements d'enseignement et de recherche français ou étrangers, des laboratoires publics ou privés. 


\title{
Finding Maximal Common Subgraphs via Time-Space Efficient Reverse Search
}

\author{
Alessio Conte ${ }^{1}$, Roberto Grossi ${ }^{2}$, Andrea Marino $^{2}$, and Luca Versari ${ }^{2}$ \\ 1 National Institute of Informatics, Tokyo, Japan, conte@nii.ac.jp \\ 2 Università di Pisa, Pisa, Italy, \{grossi, marino, luca.versari\}@di.unipi.it
}

\begin{abstract}
For any two given graphs, we study the problem of finding isomorphisms that correspond to inclusion-maximal common induced subgraphs that are connected. While common (induced or not) subgraphs can be easily listed using some well known reduction and state-of-theart algorithms, they are not guaranteed to be connected. To meet the connectivity requirement, we propose an algorithm that revisits the paradigm of reverse search and guarantees polynomial time per solution (delay) and linear space, on top of showing good practical performance.
\end{abstract}

\section{Introduction}

The problem of finding common subgraphs, as studied in this paper, has been introduced and investigated in the practical setting of proteins [5]13]14, and can be employed to mine significant information in many domains, for example identifying compound similarity and structural relationships between biological molecules [9. These patterns find motivation in the increasing amount of structured data arising from X-ray crystallography and nuclear magnetic resonance. For these reasons, the bioinformatics community has repeatedly expressed its interest in the detection of common subgraphs (see for instance [9]12 14|21]).

From a computational point of view, the problem has been studied as one of the application examples of an algorithmic framework 7 to efficiently enumerate maximal subgraphs satisfying a given property (e.g. being a clique, a cut, a cycle, a matching, etc.), also known as set systems [16. In this paper we are interested to design efficient algorithms for the following scenario.

For any two given input graphs $H$ and $F$, a subgraph $S$ of $H$ is in common with $F$ if $S$ is isomorphic to a subgraph of $F$ : it is maximal if there is no other common subgraph that strictly contains it, and maximum if it is the largest. The maximum common subgraph problem asks for the maximum ones, or simply for their size. The maximal common subgraph (MCS) problem further requires discovering all the MCs's of $H$ and $F$. The MCS problem can be constrained to connected and induced subgraphs (MCCIS) 3[13]14, where the latter means that all the edges of $H$ between nodes in the MCS are mapped to edges of $F$, and vice versa: considering induced subgraphs reduces the search space [3, and their connectivity further alleviates the explosion of the number of solutions [1314, as otherwise each permutation of a maximal independent set corresponds to a different maximal isomorphism. 


\begin{tabular}{|c|c|c|c|c|c|c|c|c|c|c|c|c|c|}
\hline \multicolumn{3}{|c|}{ GRAPH $H$} & \multicolumn{3}{|c|}{ GRAPH $F$} & \multirow{2}{*}{$\sigma$} & \multirow{2}{*}{$q$} & \multicolumn{2}{|c|}{ Koch 13] } & \multicolumn{2}{|c|}{ BC-ENUM } & \multicolumn{2}{|c|}{ par.BC-ENUM } \\
\hline$n$ & $m$ & $\Delta_{H}$ & $n$ & $m$ & $\Delta_{F}$ & & & TIME & \#sol & TIME & \#sol & TIME & $\#$ sol \\
\hline 200 & 235 & 5 & 200 & 234 & 7 & 5 & 12 & $28 \mathrm{~s}$ & 6691 & $0.2 \mathrm{~s}$ & 6691 & $0.04 \mathrm{~s}$ & 6691 \\
\hline 100 & 122 & 7 & 100 & 119 & 5 & 4 & 22 & $11 \mathrm{~s}$ & 3654 & $0.6 \mathrm{~s}$ & 3654 & $0.1 \mathrm{~s}$ & 3654 \\
\hline 2763 & 9488 & 14 & 2629 & 9059 & 12 & 12 & 68 & $2 \mathrm{~h}$ & 1998 & $2 \mathrm{~h}$ & 33874 & $2 \mathrm{~h}$ & 887293 \\
\hline
\end{tabular}

Table 1. Comparison of polynomial space algorithms: running time of Koch's [13 algorithm vs our BC-ENUM and its parallel implementation. The first two rows are two pairs of random Erdos-Renyi graphs, and the last one a pair of graphs representing proteins from the Protein Data Bank (1ald and 1gox) with a time limit of two hours. As for the notation, $n, m$, and $\sigma$ are the number of nodes, edges, and node labels of the graphs, $\Delta_{H}$ and $\Delta_{F}$ their maximum degrees, and $q$ the size of the largest found MCCIS.

MCCIS problem. Given any two graphs $H$ and $F$, list all (isomorphisms corresponding to) maximal common connected induced subgraphs (MCCIS's) between $H$ and $F$ in polynomial time per solution and total polynomial space.

Actually, MCS and MCCIS will refer to isomorphisms corresponding to MCS or MCCIS. Note that solving the above MCCIS problem is computationally more demanding than listing just maximal common induced subgraphs (i.e. relaxing the connectivity constraint) as we will comment later in the state of the art.

Contributions. We present algorithm BC-ENUM, which lists the (isomorphisms corresponding to) MCCIS's with polynomial delay and using linear total space. Given any two graphs $H$ and $F$, let $\Delta_{H}$ and $\Delta_{F}$ be their maximum degree, respectively. For each reported MCCIs, letting $q$ be its number of nodes, we pay $O\left(q^{4} \Delta_{H}^{2} \Delta_{F}^{2}\right)$ time using $O(q)$ space: the time complexity gives the delay, which is the worst-case time between any two consecutively reported MCCIS's. Note that a strength of these bounds is that they are parameterized by the solution size $q$ and independent of the sizes of $H$ and $F$ (just their maximum degree).

Table 1 reports the running time of a sequential and parallel implementation ${ }^{3}$ of BC-ENUM in C++, compared to the state-of-the-art algorithm by Koch [13]. Experiments were executed on a 12-core machine with two Intel Xeon E5-2620 CPUs and 128 gigabytes of RAM, with a time limit of two hours, showing that on top of giving theoretical guarantees, BC-ENUM is also fast in practice.

Clarification on maximum vs maximal common subgraphs. As it is clear, maximal and maximum subgraphs are inherently different problems: listing all maximal ones can potentially find an exponential number of solutions, while finding the maximum connected ones corresponds to just the single largest one, and is in practice much faster (e.g. [19]). As pointed out in [511314, however, a maximum common subgraph does not always contain all the relevant/large common structures, which motivates the MCCIS problem.

${ }^{3}$ Code available at https://github.com/veluca93/parallel_enum/tree/bccliques as part of a parallel enumeration framework. 


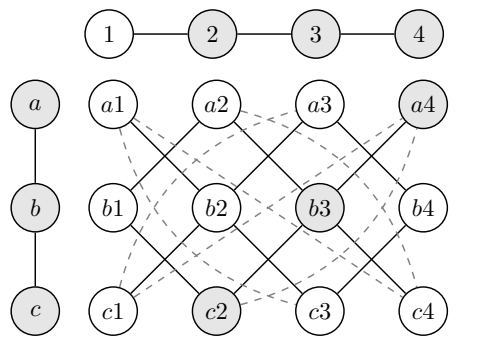

Fig. 1. An example of MCCIS ( $\{a, b, c\}$ to $\{4,3,2\}$, in this order), with the corresponding BC-clique $\{a 4, b 3, c 2\}$. White edges are represented as dashed lines.

Converting the MCCIS problem to a maximal clique problem. Cliquebased methods are widely employed on the product graph $G$, which transforms common subgraphs of $H$ and $F$ into maximal cliques in $G$, as proved in [17.

As in [13, we define the product graph between $H$ and $F$ as follows. (i) any pair of nodes $(x, i) \in H \times F$ is a node of $G$ iff they have the same label; (ii) there is a black edge between $(x, i)$ and $(y, j)$ iff $(x, y) \in E(H)$ and $(i, j) \in E(F)$; (iii) there is a white edge between $(x, i)$ and $(y, j)$ iff $x \neq y, i \neq j,(x, y) \notin E(H)$ and $(i, j) \notin E(F)$, where $E(\cdot)$ denotes the edge set.

The key property is that MCCIS's between $H$ and $F$ correspond to cliques in $G$ spanned by black edges [13, which we will call BC-cliques. An example is shown in Fig. 1.

Role of the reverse search. Reverse search is a powerful enumeration technique, introduced by Avis and Fukuda [1, that applies to a wide range of problems (e.g. [5]18). If we try to apply it to BC-cliques, a number of obstacles appear along the road and thus this paper proposes a novel, restructured, way to use reverse search on BC-cliques: Cao et al. 3] observe that materializing the product graph $G$ can be memory-wise expensive. BC-ENUM does not materialize $G$, but navigates the huge solution space of the BC-cliques by navigating $G$ implicitly using $H$ and $F$, just requiring $O(q)$ additional space (e.g. for $H$ and $F$ in the last row of Table 1. $G$ would contain millions of nodes whereas $q=68$ ). This simultaneously improves memory usage and running time, as detailed in Section 5 .

State of the art and related work. Common subgraphs problems have been studied for decades [310], with the great majority of the results dealing with maximum common subgraphs, rather than MCCIS's as we do. Previous work can be roughly classified into the following categories: backtracking methods [15, techniques based on special classes of graphs [11], clique-based methods [10[14]19], methods which are applications of a generic framework 4, and restricted to trees [8]. Among of them, Koch [13] considers MCCIS's and employs a modified version of the Bron-Kerbosch algorithm [2] to work on explicit product graphs: it is still the state of the art [22, greatly used in practice, even in the very last years (e.g. 23). Further methods have relaxed the definition of MCCIS to improve the practical performance, at the price of loosing some solutions [5]. Unfortunately, the aforementioned algorithms, when applied to listing all the 
MCCIS's, do not give any guaranteed polynomial bound on space or time per solution. Interestingly, a couple of other roads can be pursued successfully.

The framework presented in [4] uses the formulation of the reverse search on restricted problems and introduces new techniques for a class of set systems satisfying the connected hereditary property. The space is proportional to the number of solutions found, so it can be exponential, and thus space efficiency is one of the open problems posed there.

Along these lines, the framework presented in 7 provides new techniques for the class of set systems called commutable, and requires total polynomial space independently of the number of solutions found, thus answering to the question posed in 4 .

We observe that BC-cliques can fit both frameworks, with polynomial time per solution. In this paper, we focus on the latter to provide polynomial bounds on the delay and space, while the implementation of the former in practice deserves further investigation in future work to evaluate the impact of the higher space usage. Compared to the bounds polynomial in the graph size from [7, which is a general theoretical framework for which BC-cliques are just an instance, BC-ENUM aims at specializing and parameterizing these bounds for the MCCIS problem, so they are polynomial in the max degree of the graphs (rather than in the size of the product graph), and at providing practical performance.

\section{Using Reverse Search for Finding BC-cliques}

As in [13, we reduce the problem of finding MCCIS's to finding BC-cliques in the product graph. Hence, in this section, we focus on the problem of listing BC-cliques in a graph $G$, whose edges are colored black or white, where a BC-clique is a maximal clique whose black edges connect all the nodes. To this aim, we employ reverse search, which can be successfully used when a suitable parentchild relationship between solutions is defined (see for instance [1]6[18]). Here we restructure the technique to deal with the more challenging BC-cliques. We keep the schema very simple for the sake of description, and hide the technical complexity in the definition of the parent-child relationship between solutions, which is the difficult part and it will be described in the following sections.

General Scheme. As is the case of reverse-search algorithms, our algorithm will implicitly define a rooted forest among all solutions, where some solutions are the roots, and from each solution we can find all its children in the forest. As we can identify all the roots and recursively visit the children of each solution, Algorithm 1 will not miss any solution.

In the following, let $P$ be the parent of a solution $S$, denoted as $\mathrm{P}(S)$, if $S$ is a child of $P$ in this rooted forest-like structure. Note that every solution has exactly one parent, except the roots who have none.

Lemma 1. Algorithm 1 lists all maximal BC-cliques when the following conditions are all met. 


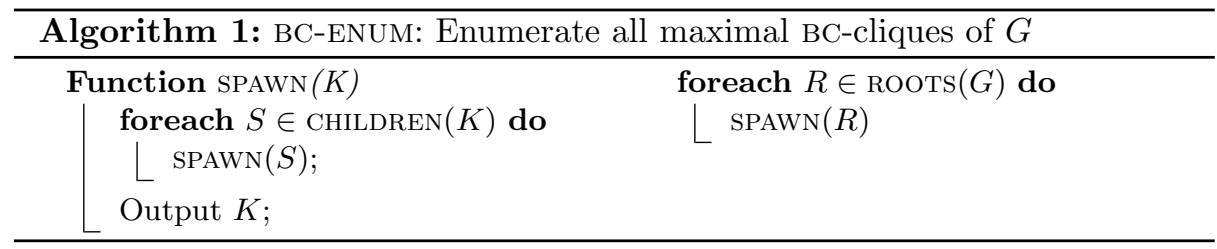

1. Each solution is either a root, or has exactly one parent.

2. The edges $\mathrm{P}(S) \rightarrow S$ induce a forest $\mathcal{Z}$ whose sources are the roots.

3. The generic function CHILDREN $(P)$ computes the set $\{S: \mathrm{P}(S)=P\}$.

The proof of Lemma 1 is straightforward as Algorithm 1 corresponds to a recursive traversal of the trees composing the forest $\mathcal{Z}$ induced by the parent-child relationship. We will design the latter so that the properties in Lemma 1 are satisfied. We remark that a tree traversal, rather than a graph traversal, does not require keeping track of visited nodes so far, and can be done without storing any information other than the current node and the previously visited one. We use this property to define an equivalent algorithm, which we call "stateless", that uses just $O(q)$ space and has the same complexity. We give further discussion in Section 5, and refer the reader to [6].

\section{Canonical Representation and Operations}

We consider $G$ to have an arbitrary ordering of the nodes $\left\langle v_{1} \ldots v_{n}\right\rangle$, and we consider each node $v_{i}$ to have label $i$. A node $v_{i}$ is smaller than $v_{j}$ if $i<j$. For convenience, we call $G_{B}$ the subgraph of $G$ induced by the black edges. We introduce the representation for BC-cliques in $G$ at the base of our approach. For a BC-clique $K$, we call the smallest node in $K$ the head of $K$, and define the notion of black-edge distance as follows.

Definition 1 (black-edge distance). The black-edge distance $\beta_{K}(v)$ of a node $v \in K$ is the distance in the induced subgraph $G_{B}[K]$ between $v$ and the head of $K$. If $v \notin K$ but $K \cup\{v\}$ is a BC-clique, $\beta_{K}(v)$ is similarly defined on $G_{B}[K \cup\{v\}]$.

When $K$ is clear from the context, we will omit the subscript and just write $\beta(v)$. (When $v$ is the head, $\beta(v)=0$.) Moreover, let us define the canonical order of $K$.

Definition 2 (canonical order). Given a BC-clique $K$, the canonical order of $K$ is $\left\langle k_{1}, \ldots, k_{|K|}\right\rangle$, where elements of $K$ are ordered in increasing lexicographical order of the pairs $\left(\beta\left(k_{i}\right), k_{i}\right)$.

This order is a specialized version of the layer-based canonical order used in [7], which is the key to bound the running time to parameters of the two original input graphs $H$ and $F$, rather than that of the larger $G$. 

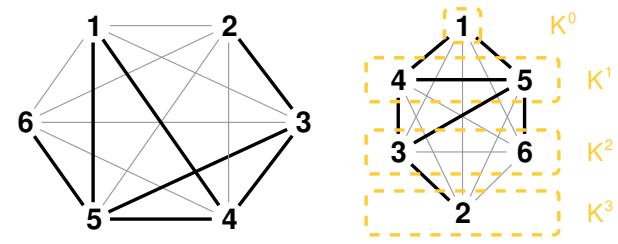

Fig. 2. A BC-clique $K$ (left) and its canonical form $K^{0} \ldots K^{3}$ (right)

We will also refer to $K^{i}$ as the set of nodes $v$ with $\beta(v)=i$. This order essentially corresponds to the visiting order of a breadth-first search of $G_{B}[K]$, starting from the head $k_{1}$, where ties in the distance from $k_{1}$ are broken by taking the node with smallest label. As prefixes will be used extensively in our approach, we define $K_{<k_{i}}$ as the prefix $k_{1}, \ldots, k_{i-1}$ of $K$. It can be easily seen from the above how any prefix of $K$ is a (non maximal) BC-clique.

An example of a BC-clique $K$ in canonical order is shown in Fig. 2 with $K^{i}$ 's ordered by node label, where the head is node 1 and, for instance, $\beta_{K}(3)=2$. When levels are relevant in the context, we represent $K$ as a sequence of sets, which corresponds to the $K^{i}$ 's in increasing order: the clique in the example would be represented as $K=\{1\},\{4,5\},\{3,6\},\{2\}$. As an example of prefix we have $K_{<3}=\langle 1,4,5\rangle$

We define the lexicographical order on BC-cliques using our canonical form. For any two pairs of integers $(a, b)$ and $(c, d)$, we write $(a, b)<(c, d)$ if the former pair is lexicographically smaller than the latter.

Definition 3 (lexicographical order). Given two distinct maximal BC-cliques $K$ and $J$, in their canonical orders $\left\langle k_{1}, \ldots, k_{|K|}\right\rangle$ and $\left\langle j_{1}, \ldots, j_{|J|}\right\rangle$, we say that $K$ is lexicographically smaller than $J$, denoted as $K<J$, iff $\left(\beta_{K}\left(k_{i}\right), k_{i}\right)<$ $\left(\beta_{J}\left(j_{i}\right), j_{i}\right)$, where $i$ is the smallest index for which $\left(\beta_{K}\left(k_{i}\right), k_{i}\right) \neq\left(\beta_{J}\left(j_{i}\right), j_{i}\right)$.

We also define a forced order of $K$ with respect to $x \in K$, which is obtained by the same process as the canonical ordering, but computing the black-edge distances $\beta$ with respect to $x$ rather than the head of $K{ }^{4}$ A prefix of $K$ with respect to $x$ corresponds to a prefix of the forced order of $K$ with respect to $x$. Clearly, this kind of prefix also corresponds to a BC-clique.

We now introduce the function COMPLETE(), which will be a key component as in 6[18. Given a BC-clique $K^{\prime}$ which may or may not be maximal, COMPLETE $\left(K^{\prime}\right)$ returns a maximal BC-clique $K$ such that $K^{\prime} \subseteq K$. This is achieved by recursively and greedily adding to $K^{\prime}$ the node $x \notin K^{\prime}$ that minimizes $\left(\beta_{K^{\prime}}(x), x\right)$, among all $x$ for which $K^{\prime} \cup\{x\}$ is a BC-clique. It is important to notice that the head of $K^{\prime}$ changes (as well as the values of $\beta_{K^{\prime}}()$ ) whenever an element with label smaller than the current head is added. The COMPLETE operation is detailed in Algorithm 2. Finally, we remark that the properties of the COMPLETE function defined in [6], that make the reverse search algorithm work for cliques, are NP-hard to obtain in the context of BC-cliques [7].

\footnotetext{
${ }^{4}$ Thus the forced order of $K$ with respect to its head is indeed the canonical order.
} 


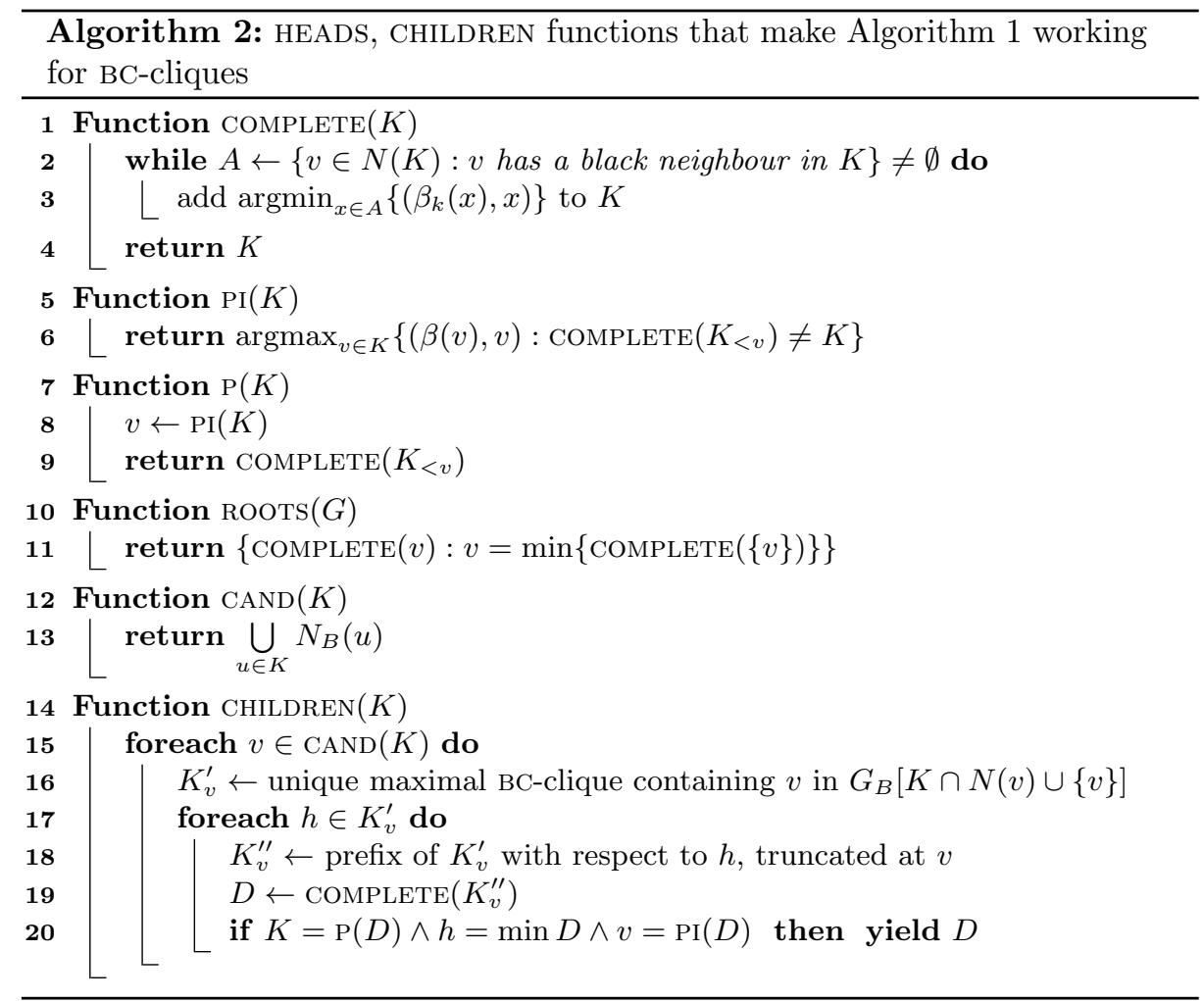

\section{Main Algorithm}

In this section we describe the proposed algorithm BC-ENUM, using the definitions given in Section 3 , to obtain the desired reverse search structure described in Section 2

Firstly, using the function COMPLETE() we can easily identify the solutions which will be the roots of the forest $\mathcal{Z}$ induced by the parent-child relationship.

Definition 4 (root). Let $K$ be a maximal BC-clique and $h=\min K$ its head. Then, $K$ is a root if and only if COMPLETE $(\{h\})=K$.

This definition implies that the number of roots is at most $n$, and each can be identified by performing COMPLETE $(v)$ on some node $v$. We now give definitions of $\mathrm{P}$ and CHILDREN as detailed in Algorithm 2.

The parent $\mathrm{P}(K)$ of $K$ is defined as the result of applying COMPLETE to the longest prefix $K_{<v}$ such that this operation does not yield $K$. We call the element $v$ of $K$ that immediately follows this prefix $K_{<v}$ the parent index of $K, \operatorname{PI}(K)$. The definitions of $\mathrm{P}$ and root are consistent with Definition 4 .

Lemma 2. $\mathrm{P}(K)=$ NULL if and only if $K \in$ ROOTS. 
We give a definition of CHILDREN, whose correctness will be proven in Section 4.1. Given a BC-clique $K$, let $\operatorname{CAND}(K)$ be the set of nodes that do not belong to $K$, but are neighbors of some node of $K$ in $G_{B}$. For each such node $v$, we compute the largest BC-clique $K_{v}^{\prime}$ that contains $v$ and is contained in $K \cup\{v\}$ (which corresponds to the connected component containing $v$ in $G_{B}[K \cap N(v)]$ ). Then, for each $h \in K_{v}^{\prime}$, we consider the forced order $\left\langle k_{1}, \ldots, k_{\left|K_{v}^{\prime}\right|}\right\rangle$ with respect to $h$ (noting that $k_{1}:=h$ ), and compute $K_{v}^{\prime \prime}$ as the prefix $k_{1}, \ldots, k_{i}$ of this order truncated at $k_{i}=v$.

Finally, we compute $D=\operatorname{COMPLETE}\left(K_{v}^{\prime \prime}\right)$ and control if $D$ satisfies the check at line 20, which is required to ensure that the parent of $D$ is indeed $K$ and that we did not generate $D$ multiple times from $K$ itself.

\subsection{Correctness}

In order to prove the correctness of Algorithm 1 using the routines defined in Algorithm 2, we prove that the conditions listed in Lemma 1 are met, recalling that the directed graph $\mathcal{Z}$ induced by the parent function $\mathrm{P}$ has the arcs from $\mathrm{P}(K)$ to $K$ for each solution $K$. By definition of $\mathrm{P}$ and by Lemma 2 we get Condition 1 Lemma 3 focuses on Condition 2 , and Lemma 4 focuses on Condition 3.

Lemma 3. The directed graph $\mathcal{Z}$ induced by $\mathrm{P}$ is a forest rooted in RooTs.

Lemma 4. If $K=\mathrm{P}(S)$, then $S \in \operatorname{CHILDREn}(K)$.

Proof. Consider an execution of $\operatorname{CHILdRen}(K)$, referring to its implementation in Algorithm 2, and let $S$ be an arbitrary maximal BC-clique with $\mathrm{P}(S)=K$. We need to prove that at some point in the execution Algorithm 2 will choose $v=\operatorname{PI}(S)$ and $h=\min (S)$ in lines 15 and 17 respectively, and that this will give $D=S$ on line 19, which means $D$ is yielded in line 20 .

Consider the prefix $S_{<v}$ of $S$. As $K=\mathrm{P}(S)=\operatorname{Complete}\left(S_{<v}\right)$, clearly $S_{<v} \subset K$ and, since $S$ has a parent, it is not a root and so $S_{<v} \neq \emptyset$. By definition of prefix, $S_{<v} \cup\{v\}$ is a BC-clique, so there must be a black edge between $v$ and a node in $S_{<v}$, and thus to a node in $K$ since $S_{<v} \subset K$, meaning that $v \in$ CAND and $v$ is considered on line 15 .

Consider now the execution of lines 1620 when $v=\operatorname{PI}(S)$. We have that $S_{<v} \cup\{v\}$ must be a subset of $K_{v}^{\prime}$, the maximal BC-clique in $G_{B}[K \cap N(v) \cup\{v\}]$ containing $v$, since $S_{<v} \cup\{v\}$ is a BC-clique containing $v$ and contained in $K \cup\{v\}$. Since $\min (S) \in S_{<v} \subseteq K_{v}^{\prime}, h$ will be chosen as $\min (S)$ in some iteration of line 17

Finally, we need to prove that $S_{<v} \cup\{v\}$ is exactly $K_{v}^{\prime \prime}$, i.e., a prefix with respect to $h$ of $K_{v}^{\prime}$. If this were not the case, let $d$ be the earliest element in $K_{v}^{\prime}$ (according to the forced ordering with respect to $h$ ) that is not in $S_{<v} \cup\{v\}$. Then $S_{<v} \cup\{v, d\}$ is still a BC-clique (as $d$ must have a backwards black edge and all nodes before $d$ are in $\left.S_{<v}\right)$; moreover, $\left(\beta_{K_{v}^{\prime}}(d), d\right)<\left(\beta_{K_{v}^{\prime}}(v), v\right)$. Thus $d$ could be chosen by COMPlete $\left(S_{<v} \cup\{v\}\right)$, and since COMPLete $\left(S_{<v} \cup\{v\}\right)=S$, this would mean that $d$ is in $S$ and occurs before $v$ in its canonical ordering, implying $d \in S_{<v}$, which is a contradiction. 
As a result, we obtain the correctness of BC-ENUM.

Theorem 1. Algorithm 1 implemented with the methods from Algorithm 2 finds all and only maximal BC-cliques exactly once.

\section{Complexity and Implicit Product Graph}

In this section, we give the complexity of BC-ENUM, taking into account that $G$ is not a generic graph with white and black edges, but an implicit product graph between $H$ and $F$ that we do not want to materialize, whose size and features depend on $H$ and $F$.

Recall that each node of $G$ corresponds to a mapping between two nodes of $H$ and $F$. For any given $v \in V(G)$, let these nodes be respectively $v_{H} \in V(H)$ and $v_{F} \in V(F)$. Further recall that $\Delta_{H}$ and $\Delta_{F}$ are the maximum node degree in $H$ and $F$, while $\Delta_{B}$ is the maximum degree in $G_{B}$. By construction of the product graph we have $\Delta_{B} \leq \Delta_{H} \cdot \Delta_{F}$. For brevity, we define $\Delta$ as $\Delta_{H}+\Delta_{F}$. These parameters are all significantly smaller than the size of $G$, which has $|V(H)| \cdot|V(F)|$ nodes, and $O\left(|V(H)|^{2} \cdot|V(F)|^{2}\right)$ edges, either black or white.

Let $X$ be a BC-clique in $G$. We denote as $X_{H}$ and $X_{F}$ respectively the set of nodes of $H$ and $F$ mapped in $X$. We keep a dictionary between the nodes of $X$ and those of $X_{H}$ and $X_{F}$, allowing us to retrieve $v_{H}$ and $v_{F}$ from $v$, or vice versa, in $O(1)$ time ${ }^{5}$

Lemma 5. Let $X$ be a BC-clique in $G$ and $v$ a node in $V(G)$. Testing whether $X \cup\{v\}$ is a $\mathrm{BC}$-clique takes $O(\min (|X|, \Delta))$ time and $O(|X|)$ space.

Proof. As $X$ is a BC-clique in $G$, in order to check that $X \cup\{v\}$ is a BC-clique in $G$ we need to check that $\{v\}$ is connected to a node in $X$ through a black edge, and to all the others through either white or black edges. This can trivially be done in $O(|X|)$ time by checking adjacency with the nodes of $X$ one by one.

However, a faster solution is possible if we focus on the edges that are not in $G$ : for a given node $x \in X$, corresponding to a mapping between $x_{H} \in V(H)$ and $x_{F} \in V(F)$, there is no edge in $G$ between $v$ and $x$ if either $\left\{v_{H}, x_{H}\right\} \in E(H)$ and $\left\{v_{F}, x_{F}\right\} \notin E(F)$, or $\left\{v_{H}, x_{H}\right\} \notin E(H)$ and $\left\{v_{F}, x_{F}\right\} \in E(F)$. Otherwise, there is either a black or white edge between $v$ and $x$.

To check the presence of missing edges between $v$ and nodes of $X$ we can simply iterate over all $x_{H} \in N_{H}\left(v_{H}\right) \cap X_{H}$, and check that each is mapped by $X$ in a node $x_{F} \in N_{F}\left(v_{F}\right) \cap X_{F}$. Then, similarly, iterate over all $x_{F} \in N_{F}\left(v_{F}\right) \cap X_{F}$ and check that they are mapped in some $x_{H} \in N_{H}\left(v_{H}\right) \cap X_{F}$. This can be done in $O\left(\left|N_{H}\left(v_{H}\right)\right|+\left|N_{F}\left(v_{F}\right)\right|\right)=O(\Delta)$ time. If no missing edge exists then $X \cup\{v\}$ is a clique in $G$. As a byproduct, this process finds all black edges between $v$ and $X$, thus we may check at the same time that there is at least one, and thus that $X \cup\{v\}$ is a BC-clique.

${ }^{5}$ This data structure will be built at the beginning of a COMPLETE call. As building it takes $O(|X|)$ time and space, it will not affect the final complexity. 
Lemma 6. For any BC-clique $X$ in $G$, computing $\beta_{X}(v)$ for all $v \in X$ takes $O\left(|X| \cdot \min \left(|X|, \Delta_{H}, \Delta_{F}\right)\right)$ time and $O(|X|)$ space.

Proof. The values of $\beta_{X}(v)$ corresponds to their distance from the head $x$ of $X$ in $G_{B}[X]$. This can be done via a BFS of $G_{B}[X]$ rooted at $x$. As $G_{B}[X]$ has $|X|$ nodes, the trivial bound for this traversal is $|X|^{2}$. Once again, we can exploit the fact that $G$ is the product graph of $H$ and $F$ : indeed, each node $v$ of $X$ corresponds to a mapping of a node $v_{H}$ of $H$ into one node $v_{F}$ of $F$. For this reason, while $v$ can have up to $\Delta_{B}$ neighbors in $G_{B}, v_{H}$ may have at most $\left|N_{H}\left(v_{H}\right)\right|$ neighbors in $X_{H}$.

We can thus iterate on the neighborhood of $v$ in $O\left(\min \left(\Delta_{H}, \Delta_{F}\right)\right)$ time by iterating on the neighbors of either $v_{H}$ in $H$ or $v_{F}$ in $F$ and then retrieve the corresponding nodes in $X$. In total, we process $|X|$ nodes, each in $O\left(\min \left(\Delta_{H}, \Delta_{F}\right)\right)$ time, or in $O(|X|)$ time using the trivial version of the BFS. The cost follows.

Lemma 7. COMplete $(X)$ takes $O\left(q\left(q+\Delta_{B}\right) \Delta\right)=O\left(q^{2} \Delta+q \Delta_{B} \Delta\right)$ time and $O(q)$ space.

Proof. In order to perform Complete $(X)$, we iterate over all nodes that can be added to $X$, adding the lexicographically smallest, with respect to $X$ and its head $x$, first. For each node $v$ in $X$ (including those that are added during the procedure), we keep an iterator which will scan in increasing order its black neighbors. Clearly, each node must be considered after the smallest ones, and once it is considered it is either added to $X$ or discarded, thus it does not need to be considered as a candidate anymore.

Given a node $c \notin X$, that has a black neighbor in $X$, we can see that $\beta_{X}(c)=\beta_{X}(v)+1$, where $v$ is the black neighbor of $c$ in $X$ that minimizes this value. Hence, to select the lexicographically smallest node, we must first consider the black neighbors of the nodes $v$ that minimize $\beta_{X}(v)$. We thus order the nodes in a priority queue by value of $\beta_{X}(v)$, breaking ties by the value of the smallest black neighbor yet to consider, so that the first node in the priority queue is the smallest candidate to consider for addition to $X$.

As $X$ will contain $|X|=O(q)$ nodes, and we will iterate on the $O\left(\Delta_{B}\right)$ black neighbors of each node exactly once, the total cost of this iteration is $O\left(q \Delta_{B}\right)$ time, and will yield up to $q \Delta_{B}$ nodes. Since by Lemma 5 testing a candidate takes $O(\min (q, \Delta))$ time, the total cost is $O\left(q \Delta_{B} \min (q, \Delta)\right)$.

Furthermore, we need to account for the cost of changing heads: after we add a node $x$ to $X$, this becomes the new head of $X$ if its label is smaller than that of the previous head. In this case, we need to update both the values of $\beta_{X}(v)$, and the priority queue of candidate nodes. By Lemma 6, this can be done in $O\left(q \min \left(q, \Delta_{H}, \Delta_{F}\right)\right)$ time. We pay this cost at most $q$ times as we add up to $q$ nodes, for a cost of $O\left(q^{2} \min \left(q, \Delta_{H}, \Delta_{F}\right)\right)$ which is upper bounded by $O\left(q^{2} \Delta\right)$.

The total cost is thus $O\left(q \Delta_{B} \min (q, \Delta)+q^{2} \Delta\right)=O\left(q^{2}\left(\Delta_{B}+\Delta\right)\right)$ time.

Lemma 8. $\operatorname{Children}(K)$ takes $O\left(q^{4} \Delta_{B}\left(\Delta_{B}+\Delta\right)\right)=O\left(q^{4} \Delta_{H}^{2} \Delta_{F}^{2}\right)$ time and $O(q)$ space. 
Proof. The cost of $\operatorname{children}(K)$ is bounded by the cost of lines 19 and 20 , times the number of nodes $\operatorname{CAND}(K)$, times the number of nodes in $K_{v}^{\prime}$.

Nodes in $\operatorname{CAND}(K)$ are at most $|K| \Delta_{B}=O\left(q \Delta_{B}\right)$ (line 13 of Algorithm 2) and $K_{v}^{\prime}$ size is bounded by $O(q)$. In the following, we prove that the time cost of line 20 of Algorithm 2 is $O\left(q\left(q+\Delta_{B}\right) \Delta\right)$. Let $\left\langle d_{1}, \ldots, d_{|D|}\right\rangle$ be the canonical ordering of $D$. By definition, $d_{i}=\operatorname{PI}(D)$ is the latest element in the canonical order of $D$ such that COMPlete $\left(D_{<d_{i}}\right) \neq D$. By the proof of Lemma 3 , we have that Complete $\left(D_{<d_{i}}\right) \leq D$ and Complete $\left(D_{<d_{j}}\right)=D$ for any $j>i$. To check that $v$ is indeed the parent index of $D$, we thus simply need to check that $\operatorname{Complete}\left(D_{<d_{i}}\right) \neq D$ and Complete $\left(D_{<d_{i+1}}\right)=D$. Furthermore, if this is the case, COMPLete $\left(D_{<d_{i}}\right)$ also gives us the parent $\mathrm{P}(D)$ of $D$. Checking that $h$ is the node of smallest label in $D$ does not affect the cost, thus the total cost is that of calling the COMPLETE() function twice. As $\Delta_{B}$ and $\Delta$ are bounded by $\Delta_{H} \cdot \Delta_{F}$, the statement follows.

Looking at Algorithm 1 1 , we can see that the complexity of BC-ENUM is bounded by the cost of the function $\operatorname{CHILDREN}(K)$. Furthermore, as shown in Lemmas 5, 6, and 7, the space required is always $O(q)$. By turning the recursion into a stateless iteration (see [6]), no more space is needed as we do not need to store the recursion stack. We also address the delay of the algorithm, that is, the maximum elapsed time between two consecutive outputs, by applying the alternative output technique in [20]: for each recursive call on $K$ in the recursion tree of Algorithm 1, we output solution $K$ at the beginning of the call if its depth is even, and at the end if it is odd. In this way, the delay is equal to the cost per solution. We can thus state the main result.

Theorem 2. Given two graphs $H$ and $F$, BC-ENUM lists all their (isomorphisms corresponding to) MCCIS's in $O\left(q^{4} \Delta_{H}^{2} \Delta_{F}^{2}\right)$ delay and $O(q)$ space.

Acknowledgements. Alessio Conte is supported by JST CREST, grant number JPMJCR1401, Japan, and Roberto Grossi, Andrea Marino and Luca Versari are supported by MIUR, Italy.

\section{References}

1. D Avis and K Fukuda. Reverse search for enumeration. Discrete Appl Math, 65(1):21-46, 1996.

2. Coenraad Bron and Joep Kerbosch. Finding all cliques of an undirected graph (algorithm 457). Commun. ACM, 16(9):575-576, 1973.

3. Y Cao, T Jiang, and T Girke. A maximum common substructure-based algorithm for searching and predicting drug-like compounds. Bioinformatics, 24(13):i366-i374, 2008.

4. S Cohen, B Kimelfeld, and Y Sagiv. Generating all maximal induced subgraphs for hereditary and connected-hereditary graph properties. J Comput Syst Sci, 74(7):1147-1159, 2008.

5. A Conte, R Grossi, A Marino, L Tattini, and L Versari. A fast algorithm for large common connected induced subgraphs. In $A l C o B$, pages 62-74, 2017. 
6. A Conte, R Grossi, A Marino, and L Versari. Sublinear-space bounded-delay enumeration for massive network analytics: Maximal cliques. In ICALP, pages 148:1-148:15, 2016.

7. A. Conte, R. Grossi, A. Marino, and L. Versari. Listing Maximal Subgraphs in Strongly Accessible Set Systems. ArXiv e-prints, March 2018. arXiv:1803.03659.

8. A Droschinsky, B Heinemann, N Kriege, and P Mutzel. Enumeration of maximum common subtree isomorphisms with polynomial-delay. In ISAAC, pages 81-93. Springer, 2014.

9. HC Ehrlich and M Rarey. Maximum common subgraph isomorphism algorithms and their applications in molecular science: a review. Wiley Interdisciplinary Reviews: Computational Molecular Science, 1(1):68-79, 2011.

10. EJ Gardiner, PJ Artymiuk, and P Willett. Clique-detection algorithms for matching 3-dimensional molecular structures. J Mol Graph Model, 15:245 - 253, 1997.

11. A Gupta and N Nishimura. Finding largest subtrees and smallest supertrees. Algorithmica, 21(2):183-210, 1998.

12. X Huang, J Lai, and S Jennings. Maximum common subgraph: some upper bound and lower bound results. BMC Bioinformatics, 7(Suppl 4):S6, 2006.

13. I Koch. Enumerating all connected maximal common subgraphs in two graphs. Theor Comp Sci, 250(1):1-30, 2001.

14. I Koch, T Lengauer, and E Wanke. An algorithm for finding maximal common subtopologies in a set of protein structures. J Comp Bio, 3(2):289-306, 1996.

15. EB Krissinel and K Henrick. Common subgraph isomorphism detection by backtracking search. Software: Practice and Experience, 34(6):591-607, 2004.

16. EL Lawler, JK Lenstra, and AHG Rinnooy Kan. Generating all maximal independent sets: NP-hardness and polynomial-time algorithms. SIAM J Comput, 9(3):558-565, 1980.

17. G Levi. A note on the derivation of maximal common subgraphs of two directed or undirected graphs. CALCOLO, 9(4):341-352, 1973.

18. K Makino and $\mathrm{T}$ Uno. New algorithms for enumerating all maximal cliques. In SWAT, pages 260-272. Springer, 2004.

19. WH Suters, FN Abu-Khzam, Y Zhang, CT Symons, NF Samatova, and MA Langston. A new approach and faster exact methods for the maximum common subgraph problem. In Computing and combinatorics, pages 717-727. 2005.

20. Takeaki Uno. Two general methods to reduce delay and change of enumeration algorithms, 2003. NII Technical Report NII-2003-004E, Tokyo, Japan.

21. RJP Van Berlo, W Winterbach, MJL De Groot, A Bender, PJT Verheijen, MJT Reinders, and D de Ridder. Efficient calculation of compound similarity based on maximum common subgraphs and its application to prediction of gene transcript levels. Int J Bioinformatics Res Appl, 9(4):407-432, 2013.

22. R Welling. A performance analysis on maximal common subgraph algorithms. In 15th Twente Student Conference on IT, The Netherlands, Un. of Twente, 2011.

23. Y Yuan, G Wang, L Chen, and H Wang. Graph similarity search on large uncertain graph databases. The VLDB Journal, 24(2):271-296, 2015. 